\title{
Gestational Diabetes Mellitus-Triage for Preventive Intervention
}

Achini Wijesinghe, ${ }^{1}$ Sonali Gunatilake, ${ }^{2}$ Dina Shrestha, ${ }^{3}$ Yashdeep Gupta, ${ }^{4}$ Noel Somasundaram, ${ }^{1}$ Uditha Bulugahapitiya, ${ }^{2}$ and Sanjay Kalra ${ }^{5}$

1. Department of Endocrinology, National Hospital of Sri Lanka, Sri Lanka; 2. Department of Endocrinology, Colombo South Teaching Hospital, Colombo, Sri Lanka; 3. Department of Endocrinology, Norvic Hospital, Kathmandu, Nepal; 4. Department of Endocrinology, All India Institute of Medical Sciences, New Delhi, India; 5. Department of Endocrinology, Bharti Hospital, Karnal, India

https://doi.org/10.17925/USE.2016.12.02.99

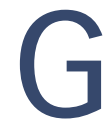
estational diabetes mellitus (GDM) is a heterogeneous condition, as exemplified by our inability to agree upon screening and diagnostic criteria. Not all women with GDM carry the same long-term risk of diabetes. We therefore propose a triage system to identify women with GDM who are at higher risk of converting to diabetes mellitus, in a shorter time frame after pregnancy. Such women can be offered personalized risk assessment information.

\section{Keywords}

Gestational diabetes mellitus, GDM, triage, postpartum, diabetes

Disclosure: Achini Wijesinghe, Sonali Gunatilake, Dina Shrestha, Yashdeep Gupta, Noel Somasundaram, Uditha Bulugahapitiya, and Sanjay Kalra have nothing to disclose in relation to this article. No funding was received for the publication of this article.

Compliance with Ethics: This article involves a review of the literature and did not involve any studies with human or animal subjects performed by any of the authors.

Authorship: All named authors meet the International Committee of Medical Journal Editors (ICMJE) criteria for authorship of this manuscript, take responsibility for the integrity of the work as a whole and have given final approval to the version to be published.

open Access: This article is published under the Creative Commons Attribution Noncommercial License, which permits any noncommercial use, distribution, adaptation, and reproduction provided the original author(s) and source are given appropriate credit.

Received: November 3, 2016

Accepted: December 12, 2016

Citation: US Endocrinology, 2016;12(2):99-101

Corresponding Author: Yashdeep Gupta, Room No 308, Biotechnology building, Department of Endocrinology \& Metabolism, AllMS, New Delhi 110029, India. E: yash_deep_gupta@yahoo.co.in
Women with gestational diabetes mellitus (GDM) carry a multidimensional and trans-generational impact. '. It poses a huge medical and public health burden on society today, which can be mitigated if appropriate proactive and preventive strategies are put in place. These include early screening and identification of women with GDM, provision of appropriate non-pharmacological and pharmacological therapy, and regular follow up after delivery to detect and treat diabetes in a timely manner. GDM is similar to pre-diabetes in many ways. ${ }^{2,3}$ Though it differs because of its association with pregnancy, the pathophysiologic features are the same as those operating in impaired glucose tolerance. Studies have reported that women with GDM are several times more likely to develop subsequent type 2 diabetes mellitus (T2DM) compared to women without GDM, with approximately $50 \%$ developing diabetes within 10 years. ${ }^{4}$ Asian Indians stand at higher risk for earlier conversion to diabetes, compared to Caucasians. Studies from India have found high conversion rates to T2DM even within five years of delivery. ${ }^{5-8}$

This reality has made GDM screening, diagnosis, management, and postpartum follow-up an international public health priority.

\section{Triage system}

GDM is a heterogeneous condition, as exemplified by our inability to agree upon screening and diagnostic criteria. ${ }^{3}$ Not all women with GDM carry the same long term risk of diabetes. We therefore propose a triage system to identify women with GDM who are at higher risk of converting to diabetes mellitus, in a shorter time frame after pregnancy. Such women can be offered personalized risk assessment information. ${ }^{9}$ Women at higher risk should be encouraged to breastfeed their infants, ${ }^{10}$ called for relatively frequent follow-up, supported with intensive lifestyle modification advice, and prescribed preventive pharmacotherapy. The health care system can use its limited resources to focus on these high risk women, and achieve greater public health benefits with a targeted approach.

\section{Determinants of risk}

A recent meta-analysis assessed 39 relevant studies on GDM, including 95,750 women. ${ }^{11}$ Body Mass Index (BMI) (realtive risk [RR] 1.95 [95\% confidence interval (Cl) 1.60, 2.31]), family history of diabetes (RR 1.70 [95\% Cl 1.47, 1.97]), non-white ethnicity (RR 1.49 [95\% Cl 1.14, 1.94]) and advanced maternal age (RR 1.20 [95\% Cl 1.09, 1.34]) were associated with future risk of T2DM. There was an increase in risk with early diagnosis of GDM (RR $2.13[95 \% \mathrm{Cl} 1.52,3.56])$, raised fasting glucose (RR 3.57 [95\% Cl 2.98, 4.04]), increased glycated hemoglobin $\left(\mathrm{HbA}_{1 \mathrm{c}}\right)(\mathrm{RR} 2.56[95 \% \mathrm{Cl} 2.00,3.17])$ and use of insulin (RR 3.66 [95\% Cl 2.78, 4.82]). Multiparity (RR 1.23 [95\% Cl 1.01, 1.50]), hypertensive disorders in pregnancy (RR 1.38 [95\% Cl 1.32, 1.45]) and preterm delivery (RR 1.81 [95\% Cl 1.35, 2.43]) 
Table 1: Suggested triage to identify high-risk women with gestational diabetes mellitus

\begin{tabular}{|l|l|}
\hline Preconception & $\begin{array}{l}\text { - Advanced age } \\
\text { - Overweight /obese } \\
\text { - Family history }\end{array}$ \\
\hline Antenatal & $\begin{array}{l}\text { - Multiparity } \\
\text { - Early diagnosis of GDM } \\
\text { - High fasting glucose/HbA1 } \\
\text { - High insulin requirement } \\
\text { - Obstetric complications, e.g., hydramnios, pregnancy } \\
\text { induced hypertension }\end{array}$ \\
\hline Neonatal & - Neonatal hypoglycemia \\
\hline Postnatal & $\begin{array}{l}\text { - Non-lactation } \\
\text { - Prolonged insulin requirement post delivery }\end{array}$ \\
\hline
\end{tabular}

$G D M=$ gestational diabetes mellitus; $H D A 1_{c}=$ glycated hemoglobin.

were associated with future diabetes."1 Prepregnancy obesity, excessive weight gain from prepregnancy to postpartum, and weight gain after pregnancy are associated with increased risk of T2DM after GDM. ${ }^{12,13}$ Lie et al. evaluated the effects of prepregnancy BMI and weight change from prepregnancy to postpartum on postpartum T2DM risk among Chinese women with GDM. The multivariable-adjusted hazard ratios based on different levels of prepregnancy BMI $\left(<23,23-24.9,25-29.9\right.$, and $\left.\geq 30 \mathrm{~kg} / \mathrm{m}^{2}\right)$ were $1.00,1.77,2.35$, and $6.54(p<0.001)$ for incident T2DM. Compared with women with stable weight $( \pm 3 \mathrm{~kg}$ ), those with weight gain $\geq 7 \mathrm{~kg}$ had an $86 \%$ increased risk of diabetes. ${ }^{2}$ Moon et al. evaluated the effect of weight gain on the development of T2DM after GDM in Asian women who have a relatively low BMI. A total of 418 women with previous GDM or gestational impaired glucose tolerance were recruited and underwent an oral glucose tolerance test at six weeks postpartum and annually thereafter. They observed an increased risk of incident diabetes as the tertile of BMI change increased $(8.6 \%, 12.6 \%$, and $16.9 \%, \mathrm{p}=0.039) .{ }^{13}$ Breastfeeding has long-term protective effect of lactation on the development of T2DM in women with gestational diabetes mellitus. ${ }^{14}$

Kwak et al. used a genetic risk score based on 48 genetic variants associated with diabetes and found improved prediction of T2DM in women with a history of GDM apart over clinical risk factors. ${ }^{15}$ Metabolomics signature can also predict the transition from GDM to T2DM. ${ }^{16}$

In a separate study, genetic variants in CDKN2A/2B and haematopoietically expressed homeobox gene (HHEX) were associated with early conversion of GDM to diabetes, while variants in CDKAL1 were associated with late conversion. ${ }^{9}$

The HHEX encodes a transcription factor that is involved in ventral pancreas development. The CDKN2A and CDKN2B genes encode p16INK4a and p15INK4b, respectively, both of which regulate $\beta$-cell replication. The data imply that the decreased $\beta$-cell function in early converters might be due to genetic predisposition conferred by these variants in HHEX, at least in part. The variant that was significantly associated with late conversion to T2DM was located in CDKAL1. The CDKAL1 gene encodes cyclin-dependent kinase 5 regulatory subunit-associated protein 1-like 1. This variant is also well known for its association with T2DM and decreased insulin secretion. Additionally, a recent large-scale genome wide association (GWA) study showed that a variant in this gene is significantly associated with BMI in
East Asians. It might be possible that the risk of T2DM in late converters conferred by this variant is modulated through an interaction with obesity. ${ }^{9}$

One may consider antenatal and postnatal triage to identify women with GDM who are at 'higher risk' of developing diabetes. Clinical pointers for high risk include non-white ethnicity, advanced age, overweight/obesity, family history of diabetes, diagnosis of GDM in early pregnancy, high insulin requirement during pregnancy, dyslipidemia, and history of obstetric complications such as hydramnios and pregnancy-induced hypertension (PIH). Delivery of a macrosomic baby $(>3,500 \mathrm{~g}$ at term in South Asia), with associated neonatal hypoglycemia, ${ }^{17}$ and prolonged insulin requirement post delivery,) are also suggested as clinical means of triage. While genetic analysis and metabolomics may play an important role in triage at a later stage, its use as a tool for triage cannot be supported on the basis of current evidence. ${ }^{18}$

\section{Preventive pharmacotherapy}

The American Association of Clinical Endocrinologists (AACE) recommends use of pharmacotherapy in selected persons with pre-diabetes. ${ }^{19}$ These include persons who are pre-diabetic and are overweight/obese, or have other features of metabolic syndrome. Such a strategy is encouraged as these persons are at high risk of converting to diabetes and developing complications in the future. A similar rationale can be applied to women with GDM. Women with GDM have a high risk of conversation to T2DM, ${ }_{1}^{20}$ and hence may benefit from preventive pharmacotherapy. In line with current guidelines, metformin seems the safest option for this.

It must be remembered, however, that metformin in an adjunct to, and not a substitute for, lifestyle modification. This information must be tempered by the understanding that it is extremely difficult for the young mother to find time for her own care. Usually, her medical and nutritional needs take a back seat to the demands of her child and family. ${ }^{21}$

The Troglitazone in Prevention of Diabetes (TRIPOD) was the first randomized controlled trial to examine the effectiveness of a pharmacotherapy intervention in delaying or preventing the development of T2DM among women with a history of GDM. Women at high risk for developing T2DM, based on $75 \mathrm{~g}$ oral glucose tolerance test (OGTT) postpartum, were recruited. After 28-30 months of follow-up, the authors found a significantly lower cumulative incidence of diabetes among women receiving troglitazone as compared with women who received placebo (5.4\% versus $12.1 \%){ }^{22}$ The TRIPOD trial was discontinued in between, as the drug was withdrawn from the market due to reports of hepatotoxicity. In Diabetes Prevention Program (DPP) study, metformin also resulted in significant reduction of about 50\% in incidence of T2DM as compared to placebo among women with GDM. ${ }^{23}$ However, adequate work has not been done to support universal use of preventive pharmacotherapy in this population. Doing so would increase the number of individuals receiving pharmacotherapy to unmanageable proportions. The health care system is not equipped to handle such a large burden of patients. Hence, the role of triage becomes even more important.

\section{Postpartum screening}

Clinical trials provide evidence that lifestyle modification as well as pharmacological intervention can prevent progression to T2DM in women with a history of GDM. There is also robust evidence that these interventions are as effective in GDM women as in people with prediabetes. Effective interventions, however, are possible only if high risk individuals are identified. This, in turn, needs rigorous postpartum follow- 
up. There has been debate on which test needs to be implemented for increasing postpartum screening rates. It should be kept in mind that the $75 \mathrm{~g}$ oral glucose tolerance test remains the gold standard. Fasting glucose estimation may miss $30-40 \%$ of cases of T2DM, and will not detect isolated impaired glucose tolerance. $\mathrm{HbA}_{1 \mathrm{c}}$ as a screening test has not been adequately studied..$^{24}$ In Asian populations, Fasting plasma glucose (FPG) and $\mathrm{HbA}_{1 \mathrm{c}}$ concentrations have much lower sensitivity than postprandial glucose concentration for detection of diabetes. In the DECODA study (a study of 11 Asian cohorts), more than half of patients with diabetes had isolated postprandial hyperglycaemia. ${ }^{25}$ We feel that postpartum women with a history of GDM should be informed that OGTT is the most sensitive screening test. In cases of lack of acceptance or practical infeasibility of OGTT, FPG or $\mathrm{HbA}_{1 \mathrm{c}}$ can be used for postpartum screening. ${ }^{26}$ Normoglycaemic women can undergo periodic screening annually whereas it may be appropriate to screen prediabetic women every six months, especially in high-risk ethnicities. However, the evidence on appropriate screening frequency is lacking. Certain populations like those with history of polycystic ovary syndrome (PCOS) and recurrent history of GDM are at high risk and should be followed closely.

\section{Summary}

Our aim is not to add controversy to the already confusing area of GDM screening and follow up. Rather, we hope to stimulate research to identify high risk women with GDM, and provide appropriate intervention to reduce their chances of converting to diabetes and developing complications. This proactive approach should create synergy of purpose amongst all health care providers involved in the care of GDM, and facilitate more efficient use of limited resources to achieve optimal public health outcomes. $\square$
1. Mahtab H, Pathan MF, Ahmed T, et al., The Dhaka Declaration 2015, Indian J Endocrinol Metab, 2015; 19:441

2. Langer O, Umans JG, Miodovnik M, The proposed GDM diagnostic criteria: a difference, to be a difference, must make a difference, I Matern Fetal Neonatal Med, 2013;26:111-5.

3. Gupta Y, Kalra B, Baruah MP, Singla R, Kalra S, Updated guidelines on screening for gestational diabetes, Int J Womens Health 2014:7:539-50.

4. Bellamy L, Casas JP, Hingorani AD, Williams D, Type 2 diabetes mellitus after gestational diabetes: a systematic review and metaanalysis, Lancet, 2009;373:1773-9.

5. Gupta Y, Kapoor D, Desai A, et al., Conversion of gestational diabetes mellitus to future Type 2 diabetes mellitus and the predictive value of $\mathrm{HbA} 1 \mathrm{c}$ in an Indian cohort, Diabet Med, 2016:[Epub ahead of print]

6. Krishnaveni GV, Hill JC, veena SR et al Gestational diabetes and the incidence of diabetes in the 5 years following the index pregnancy in South Indian women, Diabetes Res Clin Pract, 2007;78:398-404

7. Kale SD, Yajnik CS, Kulkarni SR, et al., High risk of diabetes and metabolic syndrome in Indian women with gestational diabetes mellitus, Diabet Med, 2004;21:1257-8.

8. Mahalakshmi MM, Bhavadharini B, Kumar M, et al., Clinical profile, outcomes, and progression to type 2 diabetes among Indian women with gestational diabetes mellitus seen at a diabetes center in south India, Indian J Endocrinol Metab, 2014:18:400-6.

9. Kwak SH, Choi SH, Jung HS, et al., Clinical and genetic risk factors for type 2 diabetes at early or late post partum after gestational diabetes mellitus, J Clin Endocrinol Metab, 2013;98:E744-52

10. Kalra B, Gupta Y, Kalra S. Breast feeding: preventive therapy for type 2 diabetes, J Pak Med Assoc, 2015;65:1134-6.

11. Rayanagoudar G, Hashi AA, Zamora J, Khan KS, Hitman GA Thangaratinam S, Quantification of the type 2 diabetes risk in women with gestational diabetes: a systematic review and metaanalysis of 95,750 women, Diabetologia, 2016;25:1-9.

12. Liu H, Zhang C, Zhang S, et al., Prepregnancy body mass index and weight change on postpartum diabetes risk among gestationaldiabetes women, Obesity (Silver Spring), 2014;22:1560-7.

13. Moon JH, Kwak SH, Jung HS, et al., Weight Gain and Progression to Type 2 Diabetes in Women With a History of Gestational Diabetes Mellitus, J Clin Endocrinol Metab, 2015:100:3548-55.

14. Ziegler AG, Wallner M, Kaiser I, et al., Long-term protective effect of lactation on the development of type 2 diabetes in women with recent gestational diabetes mellitus, Diabetes, 2012;61:3167-71.

15. Kwak SH, Choi SH, Kim K, et al., Prediction of type 2 diabetes in women with a history of gestational diabetes using a genetic risk score, Diabetologia, 2013:56:2556-63.

16. Allalou A, Nalla A, Prentice KJ, et al., A Predictive Metabolic Signature for the Transition From Gestational Diabetes Mellitus to Type 2 Diabetes, Diabetes, 2016:65:2529-39.

17. Retnakaran R, Glucose tolerance status in pregnancy: a window to the future risk of diabetes and cardiovascular disease in young women, Curr Diabetes Rev, 2009;5:239-44.

18. Bentley-Lewis R, Huynh J, Xiong G, et al., Metabolomic profiling in the prediction of gestational diabetes mellitus, Diabetologia, 2015;58:1329-32.

19. Garber AJ, Abrahamson MJ, Barzilay Jl, et al., AACE/ACE comprehensive diabetes management algorithm 2015, Endocrine Practice, 2015;21:438-47.

20. Bhavadharini B, Anjana RM, Mahalakshmi MM, et al., Glucose tolerance status of Asian Indian women with gestational diabetes at 6 weeks to 1 year postpartum (WINGS-7), Diabetes Res Clin Pract, 2016;117:22-7.

21. Leuridan L, Wens J, Devlieger R, Verhaeghe J, Mathieu C Benhalima K. Glucose intolerance in early postpartum in women with gestational diabetes: Who is at increased risk? Prim Care Diabetes, 2015;9:244-52.

22. Buchanan TA, Xiang AH, Peters RK, et al., Preservation of pancreatic beta-cell function and prevention of type 2 diabetes by pharmacological treatment of insulin resistance in high-risk Hispanic women, Diabetes, 2002; 51:2796-803.

23. Ratner R, Christophi $C$, Metzger B, et al., Prevention of diabetes in women with a history of gestational diabetes; effects of metformin and lifestyle interventions. I Clin Endocrinol Metab, 2008;93:4774-4779.

24. Kim C. Maternal outcomes and follow-up after gestational diabetes mellitus, Diabet Med, 2014:31:292-301

25. Qiao Q, Hu G, Tuomilehto J, et al., Age- and sex-specific prevalence of diabetes and impaired glucose regulation in 11 Asian cohorts, Diabetes Care, 2003;26:1770-80.

26. Gupta Y, Gupta A. Post-partum screening after gestational diabetes. Lancet Diabet Endocrinol, 2013;1:90-1 